\title{
Exploiting lamina terminalis appearance and motion in prediction of hydrocephalus using convolutional LSTM network
}

\author{
Görkem Saygıl11 a,b,1,*, Büşra Özgöde Yigin a, ${ }^{a}$, Gökhan Güney ${ }^{a}$, Oktay Algınn ${ }^{c, d, e}$ \\ a Department of Biomedical Engineering, Ankara University, Ankara, Turkey \\ b Department of Interdisciplinary Neuroscience, Health Science Institute, Ankara University, Ankara, Turkey \\ c Department of Radiology, City Hospital, Bilkent, Ankara, Turkey \\ d Department of Radiology, Yildirim Beyazit University, Ankara, Turkey \\ e National MR Research Center (UMRAM), City Hospital, Bilkent University, Ankara, Turkey
}

\section{A R T I C L E I N F O}

\section{Article history:}

Available online $\mathrm{xxx}$

\section{Keywords:}

MRI

Lamina terminalis

Hydrocephalus

Deep learning

ConvLSTM

CNN

\begin{abstract}
A B S T R A C T
Background. - Evaluation of the lamina terminalis (LT) is crucial for non-invasive evaluation of the CSF diversion for the treatment of hydrocephalus. Together with deep learning algorithms, morphological and physiological analyses of the LT may play an important role in the management of hydrocephalus. Aim. - We aim to show that exploiting the motion of LT can contribute to the evaluation of hydrocephalus using deep learning algorithms.

Methods. - The dataset contains 61 True-fisp data with routine sequences 37 of which are labeled as 'hydrocephalus' and the others as 'normal condition'. A fifteen-year experienced neuroradiologist divided data into two groups. The first group, 'hydrocephalus', consists of patients with typical MRI findings (ventriculomegaly, enlargement of the third ventricular recesses and lateral ventricular horns, decreased mamillo-pontine distance, reduced frontal horn angle, thinning/elevation of the corpus callosum, and non-dilated convexity sulci), and the second group contains samples that did not show any symptoms or neurologic abnormality and labeled as 'normal condition'. The region of interest was determined by the radiologist supervisor to cover the LT. To achieve our purpose, we used both spatial and spatio-temporal analysis with two different deep learning architectures. We utilized Convolutional Neural Networks (CNN) for spatial and Convolutional Long Short-Term Memory (ConvLSTM) models for spatio-temporal analysis using an ROI around LT on sagittal True-fisp images.

Results. - Our results show that $80.7 \%$ classification accuracy was achieved with the ConvLSTM model exploiting LT motion, whereas $76.5 \%$ and $71.6 \%$ accuracies were obtained by the 2D CNN model using all frames, and only the first frame from only spatial information, respectively.

Conclusion. - We suggest that the motion of the LT can be used as an additional attribute to the spatial information to evaluate the hydrocephalus.
\end{abstract}

(c) 2021 Elsevier Masson SAS. All rights reserved.

\section{Introduction}

Lamina terminalis (LT) is a thin but important sheet of the pia mater and gray matter that attaches to the superior surface of the optic chiasm (OC) and adheres to the corpus callosum by stretching the $\mathrm{OC}$, filling the gap between the $\mathrm{OC}$ and the rostrum. ${ }^{1,2}$ Monitoring of LT has been used in both the diagnosis and treatment of hydrocephalus. Previous investigations proposed that micro-

\footnotetext{
* Corresponding author at: Department of Biomedical Engineering, Ankara University, Ankara, Turkey.

E-mail address: gorkemsaygili@ankara.edu.tr (G. Saygılı).

${ }^{1}$ These authors contributed equally to this work.
}

surgical fenestration of the LT after aneurysmal subarachnoid hemorrhage (aSAH) may be correlated with a reduced rate of shuntdependent hydrocephalus ${ }^{3}$ and uses as an alternative to endoscopic third ventriculostomy (ETV) in obstructive hydrocephalus. ${ }^{4-7}$ Furthermore, some studies show that CSF hydrodynamics with brain pulsatility affect the LT motion. ${ }^{8,9}$ A preliminary study on an arguably small number of patients showed that the presence of a pulsatile LT motion by using a gated cine true fast imaging with steady-state precession (True-fisp) sequence and video tracking software can be useful for the assessment of hydrocephalus and/or ETV. ${ }^{9}$ Supporting this report, a previous study showed that morphological and physiological analyses of the LT may play an effective role in the evaluation of hydrocephalus. ${ }^{9-11}$ The most used sequence for this purpose is the Tru-fisp sequence. ${ }^{9}$ 

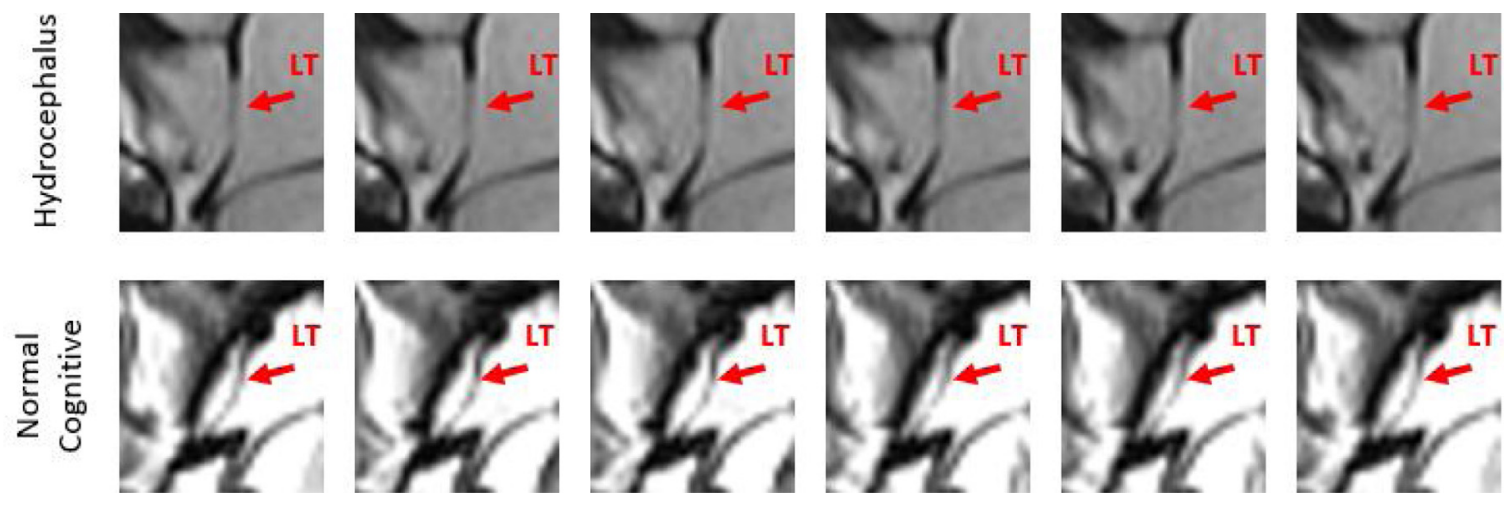

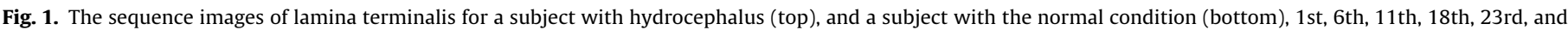
30th frames respectively.

Compared to brain lesion segmentation and tumor classification, detecting hydrocephalus is a relatively new task. Hydrocephalus has been commonly diagnosed using clinically used parameters such as Evans' Ratio, ${ }^{12}$ Cella Media Ratio, ${ }^{13}$ and Frontal Horns' Ratio. ${ }^{14}$ All these measures have been analyzed and compared in terms of accuracy, sensitivity, and specificity in a previous study. ${ }^{15}$ In another study, automatic extraction of features related to hydrocephalus has been done using segmentation. The authors proposed an algorithm that automatically measures the volume of regions with cerebrospinal fluid (CSF) and utilizes these measures as features in their support vector machine (SVM) classifier. ${ }^{16}$

Our aim of this study is to quantitatively evaluate the relationship between the LT movement and hydrocephalus using a novel, non-invasive technique. For this purpose, we trained a Convolutional Long Short-Term Memory (ConvLSTM) network to predict hydrocephalus on sagittal True-fisp images where the LT motion was visible. To our knowledge, there has not been a 3-tesla MRI study in the literature on showing the contribution of LT movement on the diagnosis of hydrocephalus using deep learning algorithms based on True-fisp data.

The rest of the paper is organized as follows: In "Methods" section, we explained the problem statement, the participants, MRI dataset, and pre-processing steps, and the models that we used as a problem-solving method. "Results" section presented the results of our experiments. We discussed our results in "Discussion" section. In "Conclusion and future work" section, we drove our conclusions and suggested possible topics for future research.

\section{Methods}

Our aim in this study is to reveal that LT movement can be used as an important feature for the evaluation of hydrocephalus. To show this, the LT region was determined as ROI, and hydrocephalus classification was made using ConvLSTM and 2D CNN models in MR scans. For this binary classification problem, we divided True-fisp data into two groups. The first group, 'hydrocephalus', consists of samples with typical symptoms and MRI findings of hydrocephalus (ventriculomegaly, enlargement of the third ventricular recesses and lateral ventricular horns, decreased mamillo-pontine distance, reduced frontal horn angle, thinning/elevation of the corpus callosum, and non-dilated convexity sulci $)^{17}$ and the second group contains samples that did not show any symptoms and labeled as 'normal condition'. Both morphology and the motion of LT differ between the subject with hydrocephalus and normal condition. But visual tracking or analysis of this movement is quite difficult, as depicted in Fig. 1. This difference can only be seen when the images are examined very carefully by experienced neuroradiolo-
Table 1

MR examination protocol of the study.

\begin{tabular}{llll}
\hline Sequence parameters & 3D-MPRAGE & 3D-SPACE & True-FISP \\
\hline TR $(\mathrm{ms})$ & 2130 & 3000 & 124.5 \\
TE $(\mathrm{ms})$ & 3.86 & 430 & 1.73 \\
FOV $(\mathrm{mm})$ & $120 \times 100$ & $174 \times 100$ & $200 \times 200$ \\
Average & 1 & 1 & 1 \\
Slice thickness $(\mathrm{mm})$ & 0.47 & 0.68 & 2 \\
Fat saturation & + & - & - \\
Distance $($ gap) & None & None & None \\
Voxel size $(\mathrm{mm})$ & $0.47 \times 0.47 \times 0.47$ & $0.68 \times 0.68 \times 0.68$ & $1.17 \times 1.17 \times 2$ \\
Number of slabs & 1 & 1 & 1 \\
Flip angle & 12 & Variant & 44 \\
Inversion time $(\mathrm{ms})$ & 1100 & $\mathrm{NA}$ & $\mathrm{NA}$ \\
Number of slices & 192 & 192 & 1 \\
Phase oversampling & $10 \%$ & $50 \%$ & - \\
\hline
\end{tabular}

Note =3D: three dimensional; W: weighted; NA: not applicable; FOV: field of view; 3D-SPACE: 3D sampling perfection with application-optimized contrasts by using different flip angle evolutions; 3D-MPRAGE: 3D magnetization-prepared rapid gradient-echo; True-FISP: 2D *tfi2d1_30 (tf2d15_retro_iPAT) sequence for lamina terminalis imaging.

gists. Hence, we used deep learning to reveal this difference from LT motion (Fig. 1).

\section{Data acquisition and participants}

This study was approved by the Human Research Ethics Committee of Ankara University (Data processing purpose, Date: 13/07/2020, Registration Number: i7-411-20); written informed consent was obtained for all patients included. The dataset contains 3-tesla retrospectively gated midline sagittal plane cine True-fisp sequence of 61 subjects ( 37 hydrocephalus, 24 normal conditions). It contains 30 male subjects between the ages of 4 and 75 and 31 female subjects aged between 13 and 81 . Obstructive (26 patients) and communicating (11 patients) hydrocephalus were present in the patient group. MRI examinations were performed using a $3 \mathrm{~T}$ unit (Trio with Tim; Siemens Healthcare AG, Erlangen, Germany) with a birdcage 32-channel head coil. Acquisition parameters of the 3-tesla MR examinations were presented in Table 1.

\section{Pre-processing}

The cine True-fisp sequence in the data set contain different numbers of frames between 11 to 50 . To prevent losing information because of this difference, all of the scans were expanded to have 50 frames with no interruption in terms of the motion. In other words, they were expanded up to 50 frames by repeating frames from the last to the first and if necessary, from first to the last without creating a discontinuity in the movement of LT. Region of interest (ROI) was determined by the experienced neuroradiologist to cover the 


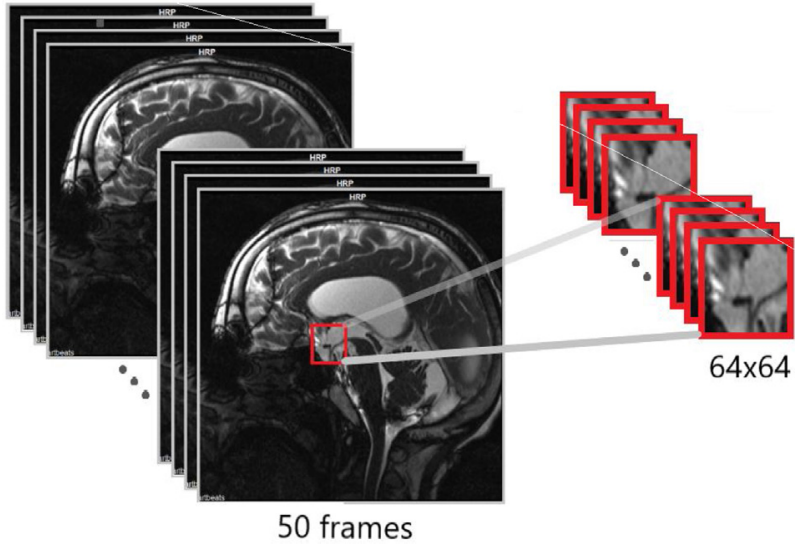

Fig. 2. Sagittal True-fisp images of a subject and cropped LT region in size $64 \times 64$.

LT (between the hypophysis and the rostrum, locating the lamina terminalis at the center). Hence, each True-fisp image was manually cropped to fit the size of $64 \times 64$ to include the LT region as seen in Fig. 2. As a result, tensors of size $50 \times 64 \times 64$ were obtained from $64 \times 64$ cropped images for each True-fisp image. The label of 1 was assigned for patients with hydrocephalus and a label of 0 for the healthy condition.

All the code used in these experiments was written using Python 3.6 and executed on Google Colab. In this environment, the Keras application program interface was utilized in conjunction with TensorFlow as a backend to build the CNN and ConvLSTM frameworks.

\section{Classifiers}

In this part, 2D CNN and ConvLSTM models were compared. For robust performance evaluation, 10 times 10 -fold cross-validation was applied for both classifiers.

\section{Convolutional Neural Network (CNN)}

Image classification with traditional machine learning algorithms requires the extraction of hand-crafted features. The performance of the traditional classifiers depends heavily on the performance of these features. In 2012, Krizhevsky et al. ${ }^{18}$ proposed a deep CNN architecture with several architectural novelties and achieved outstanding success in ImageNet classification challenge. ${ }^{19}$ Recently, CNN has become arguably the most popular deep learning architecture in medical image analysis tasks. ${ }^{20}$

The success of CNNs is mainly sourced from its capability of extracting its problem-related features. A standard CNN framework consists of two main structures. The first one consists of convolutional kernels, activation, and pooling layers and aims for automatic extraction of features from raw data. The second structure contains a fully connected multi-layer neural network that classifies from the previously learned features. ${ }^{20} \mathrm{CNNs}$ that automatically extract features from the MR images have been used to classify the images in terms of their morphological structure.

In our study, to explore the morphological changes on only spatial domain, we first applied 2D CNN only to the first frame of the True-fisp image sequences (2D CNN first frame) and then applied it to all frames and calculated the average performance (2D CNN all frames). The input size for the network was $64 \times 64$ for the cropped lamina terminalis images. In the 2D CNN model, each convolutional layer had 32 learnable filters sized as $(3 \times 3)$, as depicted in Fig. 3 . Each filter was convolved with a stride of 1 , producing 32 features maps using the same input image size of $(64 \times 64)$. The input size was halved after each pooling layer. The ReLU activation function was used in both convolution and dense layers, and the sigmoid

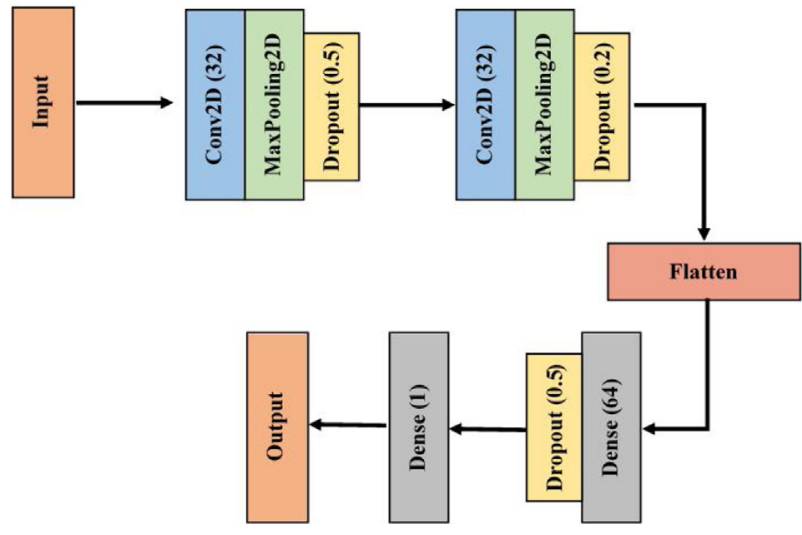

Fig. 3. 2D CNN architecture.

function was used in the last layer for the binary classification. The dropout rates used after the first and second max-pooling layers and after dense layers were set to 0.50 . The loss function was chosen as the binary cross-entropy and stochastic gradient descent (SGD) algorithm (learning rate $=0.01$, momentum $=0.0$ ) had been used as the optimizer. A total of 50 epochs were applied in the learning process (Fig. 3).

\section{Convolutional Long Short-Term Memory (ConvLSTM)}

ConvLSTM network was firstly introduced in 2015 by Shi et al. ${ }^{21}$ to exploit both temporal and spatial information inside timevarying data. ${ }^{22}$ In this network, the traditional fully connected LSTM (FC-LSTM) network was modified by using convolutional kernels between input-hidden and hidden-hidden layer connections. ${ }^{23}$ In comparison to traditional FC-LSTM, ConvLSTM is suitable for spatial sequence 3-D input. This architecture uses convolution operation instead of matrix multiplication in the LSTM cells. As a result of utilizing the power of convolution, the ConvLSTM network can find spatial features in multi-dimensional data in contrast to traditional LSTM networks. ${ }^{24}$ The equations for the ConvLSTM can be identified as follows:

$i_{t}=\sigma\left(W_{x i} * x_{t}+W_{h i} * h_{t-1}+b_{i}\right)$

$f_{t}=\sigma\left(W_{x f} * x_{t}+W_{h f} * h_{t-1}+b_{f}\right)$

$O_{t}=\sigma\left(W_{x o} * x_{t}+W_{h o} * h_{t-1}+b_{o}\right)$

$C_{t}=f_{t} \circ C_{t}+\tanh \left(W_{x c} * x_{t}+W_{h c} * h_{t-1}+b_{c}\right)$

$H_{t}=O \circ \tanh \left(C_{t}\right)$

where '*' denotes convolutional and 'o' denotes elementwise multiplications. $i_{t}, f_{t}$, and $O_{t}$ are input, forget, and output gates. $W_{x}$ stands for the weight matrix, $x_{t}, h_{t-1}$ and $C_{t}$ express current input, the output of previously hidden layer, and the state of the cell respectively. In the ConvLSTM network, filter sizes between input and hidden layers determine the shapes of formed feature maps. Similarly, for the connections between hidden layers, filter size determines the total information the received previous layers for the current time-step.

Each layer consists of 64, 32, and 16 cells, respectively. The hyperbolic tangent (tanh) function was chosen as the activation function in ConvLSTM layers and the loss function was chosen as the binary cross-entropy. A total of 25 epochs were applied in the learning process and the SGD algorithm (learning rate $=0.01$, 
G. Sayglle et al.

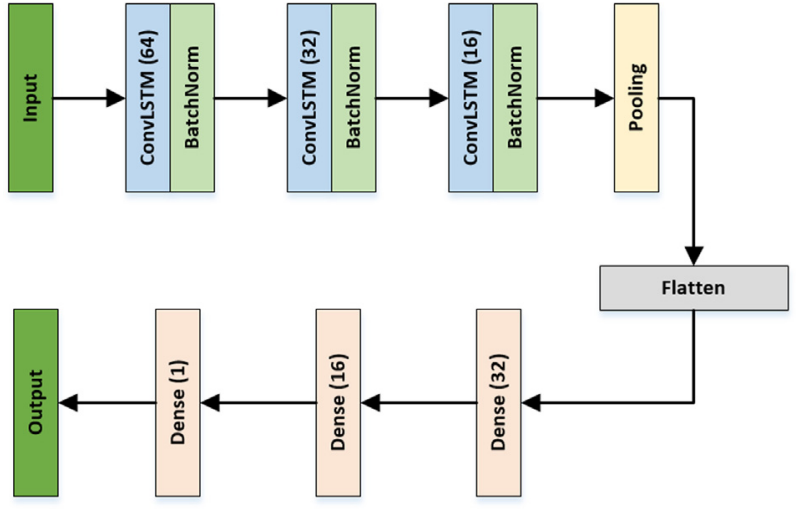

Fig. 4. Employed ConvLSTM architecture.

momentum $=0.0$ ) was chosen as the optimizer. Our ConvLSTM architecture is shown in Fig. 4.

\section{Results}

In this study, we used 2D CNN and ConvLSTM models to explore the effect of the lamina terminalis movement on predicting hydrocephalus. Using pre-processed data, all networks were fed with $50 \times 64 \times 64$ tensors size of data, and 10-times 10-fold crossvalidation was applied to show the consistency. Table 2 shows the classification results and confusion matrices are presented in Fig. 5.

As inferred from Table 2, our ConvLSTM model achieved $80.7 \%$ overall accuracy with $85.1 \%$ sensitivity and $73.8 \%$ specificity. In contrast, the 2D CNN model for all frames achieved $76.5 \%$ overall accuracy with $81.8 \%$ sensitivity and $68.3 \%$ specificity and the 2D CNN model for the first frame achieved $71.6 \%$ overall accuracy with $77.3 \%$ sensitivity and $62.9 \%$ specificity. In terms of accu-
Table 2

Classification results.

\begin{tabular}{lllll}
\hline Classifier & Accuracy & Sensitivity & Specificity & AUC \\
\hline ConvLSTM & $80.7 \%$ & $85.1 \%$ & $73.8 \%$ & $0.81 \pm 0.03$ \\
2D CNN & $76.5 \%$ & $81.8 \%$ & $68.3 \%$ & $0.76 \pm 0.01$ \\
(All & & & & \\
Frames) & & & $62.9 \%$ & $0.70 \pm 0.01$ \\
2D CNN & $71.6 \%$ & $77.3 \%$ & & \\
(First & & & & \\
Frame) & & & & \\
\hline
\end{tabular}

Note=ConvLSTM: Convolutional Long-Short Term Memory, 2D CNN: Twodimensional convolutional neural networks, AUC: Area under the curve.

racy, sensitivity, and specificity, the ConvLSTM model that explores LT movement temporarily achieved the top scores. In Fig. 6, the Receiver Operating Characteristics (ROC) curves of the networks are presented with the corresponding Area Under the Curve (AUC) scores. ConvLSTM model achieved an overall 0.81 Area Under the Curve (AUC) and the 2D CN model achieved 0.76 and 0.70 AUC scores for all frames and the first frame respectively. Following the accuracy scores, the AUC score of the ConvLSTM model was higher than the 2D CNN results for both datasets.

All experimental results favored the ConvLSTM architecture in terms of classification performance than the other two networks. These results pointed out the significance of exploiting spatial structure together with the temporal information in classifying hydrocephalus on MR images.

\section{Discussion}

In this work, we used two deep neural networks to classify hydrocephalus from normal conditions. The 2D CNN architecture was utilized to explore just the structure of the LT and excluded the temporal information from the movement of LT. LSTM can extract long term variations using states in its cells. To combine both mor-

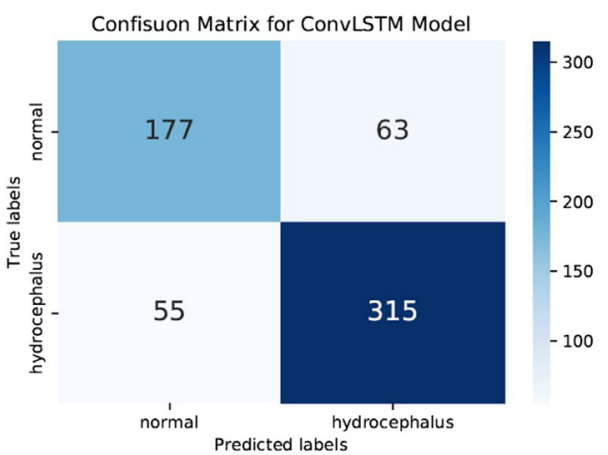

(a)

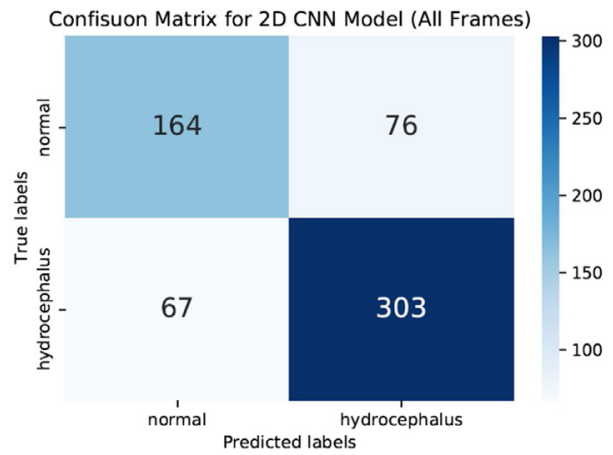

(b)

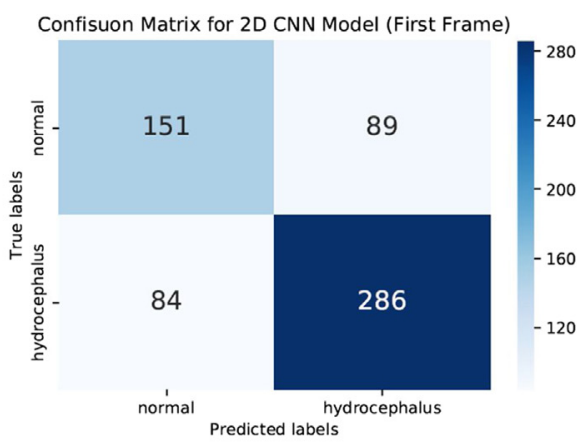

(c)

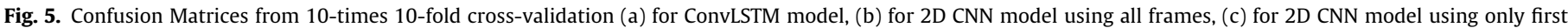
frame. 


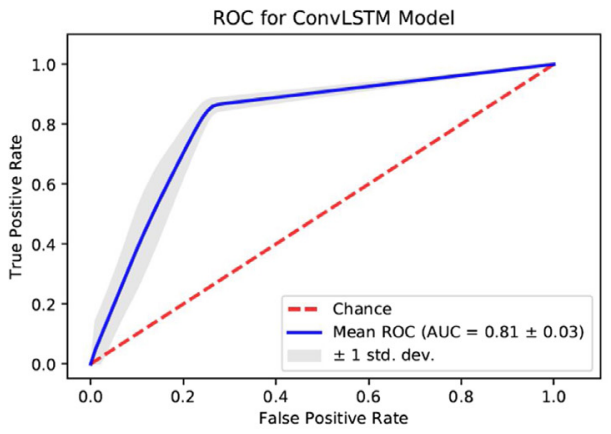

(a)

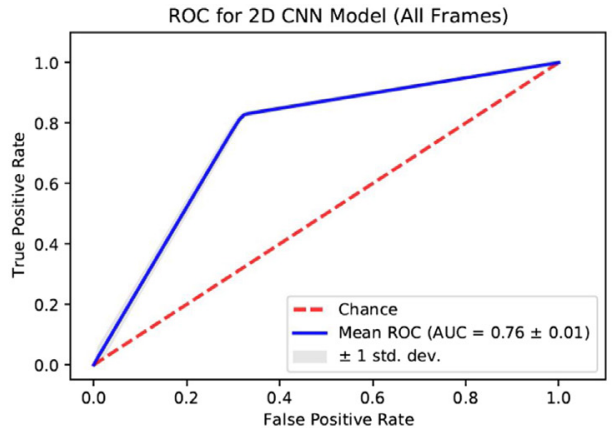

(b)

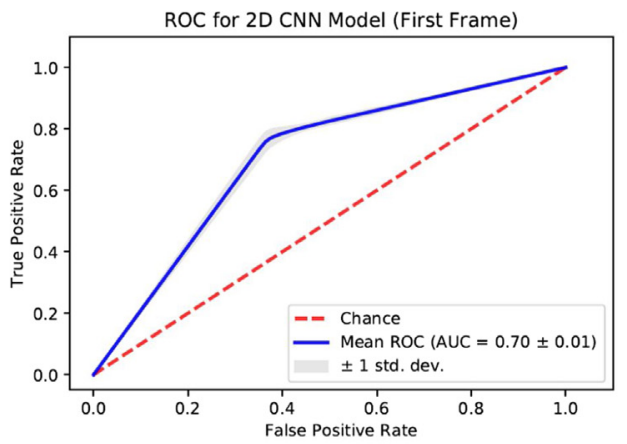

(c)

Fig. 6. ROC Curves and AUC values (a) for ConvLSTM model, (b) for $2 D$ CNN model using all frames, (c) for 2D CNN model using only first frame.

phological and temporal information in our predictions, we used a Conv-LSTM architecture. As discussed in earlier sections, ConvLSTM utilizes the capabilities of convolutional kernels from CNN for structural information and cell states from LSTM for temporal information. In our experiments, we aimed to exploit the effect of the LT motion to evaluate hydrocephalus since because of the increase of pressure in the third ventricle, the motion of LT may arguably alter compared to its motion under normal pressure. According to our experimental results, the 2D CNN (for all frames) and 2D CNN (for the first frame) models could detect hydrocephalus with $76.5 \%$ and $71.6 \%$ overall accuracy, respectively. In terms of AUC scores, the 2D CNN model achieved 0.76 and 0.70 for all frames and the first frame, respectively. Both accuracy and AUC performances showed that these two architectures can successfully identify the status of hydrocephalus with considerably high accuracy, relying mainly on structural variations without any temporal information. Compared to both results, Conv-LSTM achieved the highest scores in terms of both accuracy and AUC. This performance superiority shows the capability of Conv-LSTM in exploiting both structural and temporal variations between the two classes and suggests that the motion of the LT can be used as an important feature in the automatic evaluation of hydrocephalus. Hence, based on our results, we can conclude that the motion of LT can be used extensively in different algorithms for the automatic detection of hydrocephalus.

The superior performance of Conv-LSTM suggests that the motion of the LT is worth considering for the evaluation of hydrocephalus. These results support the conclusion of Hodel et al. ${ }^{24}$ that the quantification of the third ventricular wall movement may improve the detection of hydrocephalus. Additionally, as suggested by Algin et al., ${ }^{25}$ our results showed that the morphology of LT varies between subjects with hydrocephalus and normal conditions.

Routine morphological parameters (such as Evans index, cella media index and frontal occipital horn ratio) are practical but highly susceptible to experience of radiologist and errors related to imaging plane/level. ${ }^{26}$ Also, these indexes generally do not correlate with neurocognitive performance or intracranial pressure. ${ }^{26}$ In our study, we used the 2D-True-fisp sequence to evaluate LT motion. This sequence provides significant advantages over other sequences with its high geometric, contrast and temporal resolution values, and short acquisition time. However, the 3D- True-fisp with sub-millimetric iso-voxels may be useful to evaluate the entire LT and other ventricular membranes more globally. However, 3D imaging takes more time and decreases the temporal resolution. ${ }^{26}$ CAIPIRINHA, simultaneous multi-slice imaging, and compressedsense techniques can be useful for reducing the examination time. There is a need for prospective and comprehensive new studies in this area.

\section{Conclusion and future work}

In this work, we used a Conv-LSTM based deep learning algorithm to predict hydrocephalus using a patch around LT on the True-fisp images to explore whether LT motion variation can be used as a feature in the automatic evaluation of hydrocephalus. Our results showed that both morphology and the motion of LT can be used for the management of hydrocephalus using the True-fisp images. These results support the conclusions of previous clinical studies utilizing deep learning.

As future work, we aim to expand our dataset and use 3D volume with temporal information with an enhanced Conv-LSTM architecture on 4D data. Additionally, we plan to design a combined 3D-CNN and LSTM network to achieve higher accuracies in our predictions.

\section{References}

1. Oertel JMK, Vulcu S, Schroeder HWS, Konerding MA, Wagner W, Gaab MR Endoscopic transventricular third ventriculostomy through the lamina terminalis. J Neurosurg. 2010:113, http://dx.doi.org/10.3171/2010.6.JNS09491. 
2. Hardy DG, Peace DA, Rhoton Jr AL. Microsurgical anatomy of the superior cerebellar artery. Neurosurgery. 1980;6:10-28, http://dx.doi.org/10.1227/00006123-198001000-00002.

3. Komotar RJ, Olivi A, Rigamonti D, Tamargo RJ. Microsurgical fenestration of the lamina terminalis reduces the incidence of shunt-dependent hydrocephalus after aneurysmal subarachnoid hemorrhage. Neurosurgery. 2002;51:1403-1413, http://dx.doi.org/10. 1227/01.NEU.0000309116.09463.A6.

4. Torres-Corzo J, Rangel-Castilla L. Endoscopic third ventriculostomy. Contemp Neurosurg. 2006:28.

5. Schroeder HWS, Oertel J, Gaab MR. Endoscopic treatment of cerebrospinal fluid pathway obstructions. Oper Neurosurg. 2007;60, http://dx.doi.org/10.1227/01.NEU.0000249242.17805.E3. ONS-44-ONS-52.

6. Souweidane MM. Anterior third ventriculostomy: an endoscopic variation on a theme. J Neurosurg JNS. 2010;113:1259-1260, http://dx.doi.org/10.3171/2009.11.JNS091698.

7. Akyuz M, Tuncer R. The effects of fenestration of the interpeduncular cistern membrane arousted to the opening of lamina terminalis in patients with ruptured ACoA aneurysms: a prospective, comparative study. Acta Neurochir (Wien). 2006;148:725-732, http://dx.doi.org/10.1007/s00701-006-0738-0.

8. Reubelt D, Small LC, Hoffmann MHK, Kapapa T, Schmitz BL. MR imaging and quantification of the movement of the lamina terminalis depending on the CSF dynamics. Am J Neuroradiol. 2009;30, http://dx.doi.org/10.3174/ajnr.A1306, 199 LP-202.

9. Hodel J, Decq P, Rahmouni A, et al. Brain ventricular wall movement assessed by a gated cine MR trueFISP sequence in patients treated with endoscopic third ventriculostomy. Eur Radiol. 2009;19:2789, http://dx.doi.org/10.1007/s00330-009-1477-4.

10. Algin O. Evaluation of hydrocephalus patients with 3D-SPACE technique using variant FA mode at 3T. Acta Neurol Belg. 2018;118:169-178, http://dx.doi.org/10.1007/s13760-017-0838-z.

11. Kartal MG, Ocakoglu G, Algin O. Feasibility of 3-dimensional sampling perfection with application optimized contrast sequence in the evaluation of patients with hydrocephalus. J Comput Assist Tomogr. 2015:39.

12. Evans Jr Wa. An encephalographic ratio for estimating ventricular enlargement and cerebral atrophy. Arch Neurol Psychiatry. 1942;47:931-937, http://dx.doi.org/10.1001/archneurpsyc.1942.02290060069004.

13. Swati G, Sanjay G, Pankaj Y, Saumya M. Ct evaluation of various linear indices in children with clinically suspected hydrocephalus. J Evol Med Dent Sci. 2017;6:3078-3082.
14. Arun Kumar S, MeenaKumari S, Pavithra A, Saraswathy R. CT based study of frontal horn ratio and ventricular index in South Indian population. IOSR J Dent Med Sci e-. 2017;16:55-59, http://dx.doi.org/10.9790/0853-1607065559.

15. Ozgode Yigin B, Algin O, Saygili G. Comparison of morphometric parameters in prediction of hydrocephalus using random forests. Comput Biol Med. 2020;116:103547, http://dx.doi.org/10.1016/j.compbiomed.2019.103547.

16. Gunter NB, Schwarz CG, Graff-Radford J, et al. Automated detection of imaging features of disproportionately enlarged subarachnoid space hydrocephalus using machine learning methods. NeuroImage Clin. 2019;21:101605, http://dx.doi.org/10.1016/j.nicl.2018.11.015.

17. Kartal MG, Algin O. Evaluation of hydrocephalus and other cerebrospinal fluid disorders with MRI: an update. Insights Imaging. 2014;5:531-541, http://dx.doi.org/10.1007/s13244-014-0333-5.

18. Krizhevsky A, Sutskever I, Hinton GE. ImageNet classification with deep convolutional neural networks. Commun ACM. 2017;60:84-90, http://dx.doi.org/10.1145/3065386.

19. Deng J, Dong W, Socher R, Li L, Li K, Fei-Fei L. ImageNet: a large-scale hierarchical image database. In: 2009 IEEE Conf. Comput. Vis. Pattern Recognit. 2009:248-255, http://dx.doi.org/10.1109/CVPR.2009.5206848.

20. Litjens G, Kooi T, Bejnordi BE, et al. A survey on deep learning in medical image analysis. Med Image Anal. 2017;42:60-88, http://dx.doi.org/10.1016/j.media.2017.07.005.

21. Shi X, Chen Z, Wang H, Yeung DY, Wong WK, Woo WC. Convolutional LSTM network: a machine learning approach for precipitation nowcasting. Adv Neural Inf Process Syst. 2015, 2015-Janua:802-10.

22. Kim S, Hong S, Joh M, Song SK. DeepRain: ConvLSTM Network for Precipitation Prediction Using Multichannel Radar Data; 2017:3-6. ArXiv.

23. Medel JR, Savakis A. Anomaly Detection in Video Using Predictive Convolutional Long Short-Term Memory Networks; 2016.

24. Hodel J, Rahmouni A, Zins M, Vignaud A, Decq P. Magnetic resonance imaging of noncommunicating hydrocephalus. World Neurosurg. 2013;79, http://dx.doi.org/10.1016/j.wneu.2012.02.009. S21.e9-S21.e12.

25. Algin O, Kilın M, Ozmen E, Ocakoglu G. Assessment of liliequist membrane by 3D-SPACE technique at 3T. Neuroradiology. 2016;58:637-647, http://dx.doi.org/10.1007/s00234-016-1669-y.

26. Grimm F, Edl F, Gugel I, Kerscher SR, Schuhmann MU. Planar single plane area determination is a viable substitute for total volumetry of CSF and brain in childhood hydrocephalus. Acta Neurochir(Wien). 2020;162:993-1000, http://dx.doi.org/10.1007/s00701-019-04160-4. 